\title{
Effect of artesunate on apoptosis and autophagy in tamoxifen resistant breast cancer cells (TAM-R)
}

\author{
Xiaoqing Ding ${ }^{1}$, Wei Yue ${ }^{2}$, Haiyan Chen ${ }^{1}$ \\ ${ }^{1}$ Department of Hematology, Dongfang Hospital Affiliated to Beijing University of Traditional Chinese Medicine, Beijing 100078 , China; ${ }^{2}$ Division \\ of Endocrinology and Metabolism, University of Virginia, Charlottesville, VA 22908, USA \\ Contributions: (I) Conception and design: H Chen; (II) Administrative support: W Yue, H Chen; (III) Provision of study materials or patients: All \\ authors; (IV) Collection and assembly of data: All authors; (V) Data analysis and interpretation: All authors; (VI) Manuscript writing: All authors; (VII) \\ Final approval of manuscript: All authors. \\ Correspondence to: Haiyan Chen. Department of Hematology, Dongfang Hospital affiliated to Beijing University of Traditional Chinese Medicine, \\ No. 6 Fangxingyuan 1st Block, Fengtai District, Beijing 100078, China. Email: haiyanchenabc@126.com.
}

Background: The antitumor effect of artesunate (ART) is well-recognized. To investigate the effect of ART on tamoxifen-resistant breast cancer cells (TAM-R) proliferation, apoptosis, and autophagy with TAM-R cells of breast cancer as objects of study, and to investigate whether ART could re-sensitize TAM-R cells to TAM therapy.

Methods: Experiments were performed using TAM-R cell lines. Cell Death Detection ELISA kit was used to detect the level of apoptosis. Western blot and immunofluorescent staining analysis were conducted to detect autophagy and apoptosis related proteins in TAM-R cells.

Results: After treated with ART, the proliferation activity of TAM-R cells was inhibited in a concentrationdependent manner. Increased apoptosis activity was detected in TAM-R cells when treated with ART. Compared with $10^{-6} \mathrm{M}$ TAM monotherapy group, treatment group with ART and TAM in combination caused significant reduction in the levels of Bcl-2, XIAP, and Survivin proteins, and elevation of caspase-7. However, increased p53 proteins was not detected after ART treatment. No significant change was observed in autophagy proteins LC-3 and Beclin-1 among control, ART, TAM, and ART combined with TAM groups.

Conclusions: The intervention of ART could not inhibit protective autophagy in TAM-R cells, however, possess potential in inducing apoptosis. In addition, as ART inhibit TAM-R cells growth in a dose-dependent manner, co-administration of 1 or $3 \mu \mathrm{M}$ of ART with $10^{-6} \mathrm{M}$ TAM might not be enough to re-sensitize TAM-R cells to TAM therapy.

Keywords: Apoptosis; autophagy; artesunate; breast cancer; tamoxifen-resistant breast cancer cells (TAM-R)

Submitted Apr 04, 2019. Accepted for publication Aug 12, 2019.

doi: $10.21037 /$ tcr.2019.08.41

View this article at: http://dx.doi.org/10.21037/tcr.2019.08.41

\section{Introduction}

Breast cancer is the second highest killer of all cancers in women, causing up to $30 \%$ deaths in diagnosed patients $(1,2)$. Approximately 1.2 million new cases occur each year, and younger age patients appear more frequently than the past $(3,4)$. It is estimated that almost $70 \%$ of breast cancer patients express ER, which make endocrine therapies an important strategy (5). The "gold standard" and first-line therapy for $\mathrm{ER}^{+}$breast cancer remains to be tamoxifen (TAM). By binding to ERs, TAM can inhibit the proliferation of estrogen-stimulated breast cancer cells (6). However, acquired resistance, as a major issue, still stand in the pathway of achieving the best performance of TAM (7). Several mechanisms are responsible for acquiring TAM resistance in breast cancer cells, and accumulating 
studies have focused on inhibition of apoptosis and induction of protective autophagy $(8,9)$.

Apoptosis and autophagy are considered as two distinct processes coordinately regulating cell survival and death, and play important roles in tumor development and progression. By removing the useless, damaged, aged, and malignant cells out of the normal cells, apoptosis can help human body maintain the normal function of organs and tissue, control the tissue elements within the desired range of physiological need, stabilize the internal environment, and prevent the incidence and progression of cancer (10). Autophagy is an evolutionarily conserved process which initiated in hostile internal conditions to maintain metabolic homeostasis. It plays dual roles as destroyer or protector in cancer cells. On the one hand, autophagy can suppress tumor growth, however, it also can protect the tumor cells by delaying and limiting breast cancer cells death in response to stressor (11). Previous research considered autophagy as an important mechanism of TAM resistance, and demonstrated that resensitization of TAM resistant breast cancer cells could be achieved by inhibiting autophagy (12).

Artesunate (ART), which is a water-soluble derivative of artemisinin and an active component of the Chinese herb Artemisin annual, has been widely applied for the treatment of malarial (13). Its anticancer characteristics has also been well recognized. Back in 1996, researchers already found that ART could induce tumor cell apoptosis (14). Hereto, the potential anticancer effect of ART has manifested in an amount of tumors, including Burkitt's lymphoma, chronic myeloid leukemia, renal carcinoma, cervical cancer, breast, hand and neck cancer (15-20). Numerous mechanisms of ART on cancer cells were investigated, and reports suggested that ART inhibited proliferation of tumor cells by inducing oxidative DNA damage, apoptosis or oncosis, inhibiting angiogenesis, and arresting cell cycle (21-24). For breast cancer, studies were performed on estrogen/TAM-sensitive breast cancer cells (MCF-7), and showed promising results of ART in the inhibition of breast cancer cell proliferation by inducing apoptosis or G2/M cell cycle arrest $(19,25)$. However, no experiment has been conducted on Tamresistant breast cancer cells (TAM-R). Since majority of patients develop TAM resistance over time, finding an effective way to re-sensitized TAM-R to estrogen therapy becomes urgent. Thus, we investigated the effects of ART on apoptosis and autophagy of TAM-R cells, and evaluated whether ART could re-sensitize endocrine therapy against breast cancer after acquired resistance.

\section{Methods}

This study only involved cell lines analysis. The authors did not perform any experiments on human participants. Analysis did not involve the collection, use, or transmittal of individual identifiable data. As such, Institutional Review Board (IRB) approval to conduct this study was not required.

\section{Materials}

ART was obtained from Guilin Pharmaceutical Co., Ltd. (Guilin, China). Tamoxifen was purchased from Sigma (St. Louis, MO). Improved Minimum Essential Medium (IMEM) for cell culture was purchased from Cellgro (Pittsburgh, PA). Fetal bovine serum (FBS), glutamine, and trypsin were from Invitrogen (Carlsbad, CA). The antibodies against LC3, Beclin-1, p53, Survivin, pAMPK, $\mathrm{Bcl}-2, \mathrm{X}$-linked inhibitor of apoptosis protein (XIAP) and caspase-7 were obtained from Cell Signaling Technology (Beverly, MA). Near infra-red dye conjugated secondary antibodies for western analysis were purchased from LICOR, Inc. (Lincoln, NE). MitoTracker Red CMXRos, Hoechst33324, and Alex Fluor 488 goat anti-rabbit IgG were from Molecular Probes (Eugene, Oregon USA). Others materials were purchased from Sigma, unless indicated otherwise.

\section{Cell culture}

TAM-R cell lines derived from MCF-7 cells for breast cancer were kind gifts from Dr. Wei Yue (University of Virginia, VA, USA). Cultured medium IMEM with $5 \%$ FBS was applied for TAM-R cell cultivation $\left(37{ }^{\circ} \mathrm{C}, 5 \% \mathrm{CO}_{2}\right)$. For every 2 to 3 days, the cultured cells were passaged. TAM-R cells were cultured in TAM at a concentration of $10^{-7} \mathrm{~mol} / \mathrm{L}$ to maintain its drug resistance characteristics until one week before experiment.

\section{Determination of cell number}

TAM-R cells were seeded in six-well plates at a density of $3 \times 10^{4}-6 \times 10^{4}$ per well in IMEM supplemented with $5 \%$ FBS. Two days later, the medium was aspirated and ART or TAM at various concentrations or control were added to the wells. Treatments from day 1 were renewed on day 3 by aspirating medium from wells and replacing with fresh medium and treatments. After culture, TAM-R 
cells were washed twice with saline. Sequentially, $2 \mathrm{~mL}$ of HEPES- $\mathrm{MgCl}_{2}$ solution $(0.01 \mathrm{~mol} / \mathrm{L}$ HEPES and $1.5 \mathrm{mmol} / \mathrm{L} \mathrm{MgCl}_{2}$ ) and $0.2 \mathrm{~mL}$ of $\mathrm{ZAP}$ solution $[0.13 \mathrm{~mol} / \mathrm{L}$ ethylhexadecyldimethylammonium bromide in $3 \%$ glacial acetic acid $(\mathrm{v} / \mathrm{v})]$ were added to prepare nuclei. Cells count was performed by a model Z1 Coulter counter.

\section{Determination of apoptotic cell death}

Apoptosis was assessed using the Cell Death Detection ELISA kit (Roche Diagnostics, Indianapolis, IN) following the manufacturer's instructions. Briefly, cells were seeded into 12 -well plates at density of $8 \times 10^{4}$ per well. On day 2, testing compounds were added, and cells were cultured for desired periods of time. After cultivation, the cell was then incubated with $0.5 \mathrm{~mL}$ lysis buffer at room temperature for $30 \mathrm{~min}$ followed by centrifugation at $210 \mathrm{~g}$ for $10 \mathrm{~min}$ at $4{ }^{\circ} \mathrm{C}$ for the preparation of cell lysates. A set of parellel plates treated with the same treatment was prepared for cell counting. The results were expressed by absorbance at $405 \mathrm{~nm}$ after normalization of cell number.

\section{Western blots}

TAM-R cells were plated in $60 \mathrm{~mm}$ dishes containing $5 \%$ FBS IMEM. When cells were about $80 \%$ of confluence, they were treated with testing compound for 3 days in the same culture medium. The cultured cells were washed with phosphate buffered saline (PBS) followed by $5 \mathrm{~min}$ incubation on ice with $0.5 \mathrm{~mL}$ lysis buffer [20 mmol/L Tris (pH 7.5), $150 \mathrm{mmol} / \mathrm{L} \mathrm{NaCl}, 1 \mathrm{mmol} / \mathrm{L}$ EDTA, $1 \mathrm{mmol} / \mathrm{L}$ EGTA, $1 \mathrm{mmol} / \mathrm{L}$ sodium orthovanadate, $2.5 \mathrm{mmol} / \mathrm{L}$ sodium pyrophosphate, $1 \%$ Triton $\mathrm{X}-100$, $1 \mathrm{mmol} / \mathrm{L} \beta$-glycerophosphate, $1 \mu \mathrm{g} / \mathrm{mL}$ leupeptin and aprotinin, and $1 \mathrm{mmol} / \mathrm{L}$ phenylmethylsulfonyl fluoride (PMSF)]. Then, the cells were sonicated with pulse, and centrifuged at $14,000 \mathrm{rpm}$ for $10 \mathrm{~min}$ at $4{ }^{\circ} \mathrm{C}$. Before analysis, cell lysates were stored at $-80{ }^{\circ} \mathrm{C}$. The total protein content of the lysate was determined with a standard Bradford assay. The extracted $50 \mu \mathrm{g}$ of protein were separated on $10 \%$ Sodium dodecyl sulfate (SDS) polyacrylamide gel and transferred to a nitrocellulose membrane. The membrane was probed with primary antibodies (Cell Signaling) dissolved in Tris buffered saline (TBS) with $5 \%$ bovine serum albumin, and incubated with infra-red dye conjugated secondary antibody. Odyssey imaging scanner was used to visualized and quantified protein bands.

\section{Autophagy detection}

TAM-R cells were grown on cover slip in 6-well plates in IMEM-5\% FBS. When the cells were attached, fresh medium with treatment agents were added and incubated for $24 \mathrm{~h}$. Autophagy was detected using CytoID Autophagy Detection Kit from Enzo Life Sciences Inc. (Farmingdale, NY) following the manufacturer's instructions. Olympus IX81 microscope and SlideBook software were utilized for images taking.

\section{Statistic analysis}

All reported data were presented as means \pm SE. Statistical comparisons were conducted with two-tailed Student's $t$ tests. $\mathrm{P}$ value $<0.05$ was considered as statistical significance.

\section{Results}

\section{ART inbibits TAM-R cells growth}

TAM-R cells were treated with ART at different concentrations for 4 days before evaluation. Results from cell growth assay showed that ART inhibited the cell viability in a dose-dependent manner (Figure 1A). Upon treatment with different concentrations, a higher dose of ART was expected to have a stronger effect in the inhibition of TAM-R cells growth. We also explored whether ART could re-sensitize TAM-R cells to TAM therapy. Experiments were conducted between TAM-R cell with and without ART treatment at different TAM concentrations (Figure 1B). TAM concentration is in log scale so there is no data point of ART alone which is $50.9 \%$ of the control. The addition of TAM did not further reduce cell number in the presence of ART, suggesting that ART did not increase TAM sensitivity (Figure 1C).

\section{ART promotes apoptosis in TAM-R cells}

Although statistical significance was absent, treatment of TAM-R cells with $3 \mu \mathrm{M}$ ART, $10^{-7} \mathrm{M}$ TAM plus $3 \mu \mathrm{M}$ ART, and $10^{-6} \mathrm{M}$ TAM plus $3 \mu \mathrm{M}$ ART for 72 hours still evidently enhanced apoptosis according to the apoptosis ELISA assay (Figure 1D). To further confirm the apoptosis activity induced by ART, we assessed the levels of several key proteins related to the process of apoptosis using western blotting. Anti-apoptotic proteins Bcl-2, Survivin, and XIAP, and pro-apoptotic proteins $\mathrm{p} 53$ and caspase- 7 were assessed. As shown in Figure 2, the combination of high-dose TAM 

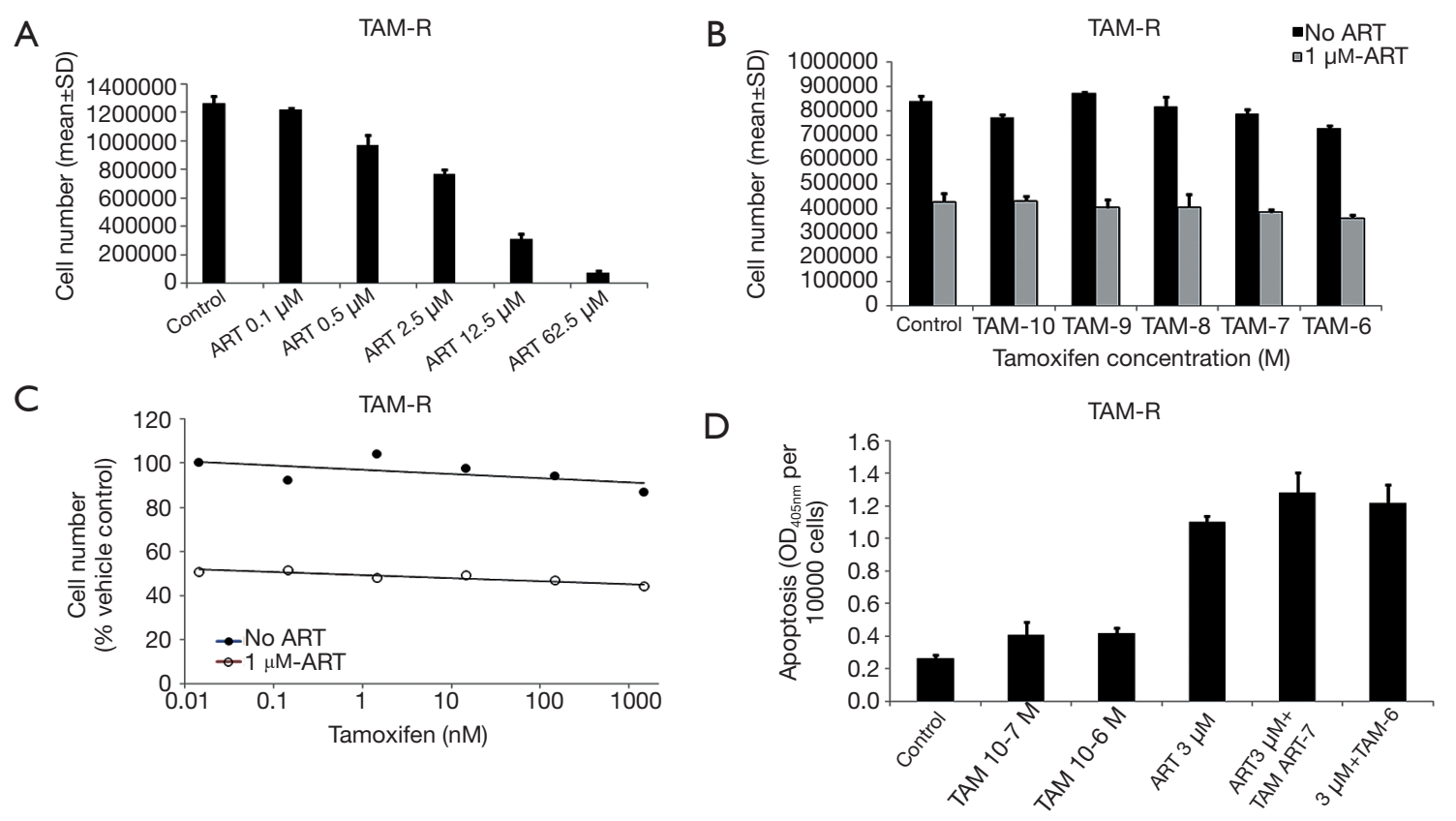

Figure 1 Effect of artesunate (ART) (4 days) on tamoxifen-resistant breast cancer cells (TAM-R) cell growth. (A) TAM-R cells were seeded in six-well plates at a density of $3 \times 10^{4}-6 \times 10^{4}$ per well in Improved Minimum Essential Medium (IMEM) supplemented with $5 \%$ fetal bovine serum (FBS) and cultured for 4 days. ART inhibited the cell viability in a dose-dependent manner; (A,C) artesunate \pm tamoxifen were seeded in six-well plates at a density of $3 \times 10^{4}-6 \times 10^{4}$ per well in IMEM supplemented with $5 \%$ FBS and cultured for 5 days. (C) is replot of the data of cell counting with or without ART (B) with regression lines. Artesunate could not re-sensitize TAM-R cells to tamoxifen treatment. (D) TAM-R cells with different treatment options were seeded in six-well plates at a density of $3 \times 10^{4}-6 \times 10^{4}$ per well in IMEM supplemented with $5 \%$ FBS and cultured for 3 days. Artesunate promotes apoptosis in TAM-R cells.

with ART treatment significantly reduced Bcl-2, Survivin, and XIAP levels compared with $10^{-6} \mathrm{M}$ TAM treatment alone. Also, higher dose of ART appeared to have better effect in inhibiting these anti-apoptotic proteins. ART increased the protein expression of caspase-7, which is an executioner protein of apoptosis. The combination used of $3 \mu \mathrm{M}$ ART plus $10^{-6} \mathrm{M}$ TAM showed to have the strongest effect on caspase-7. For $\mathrm{p} 53$, another pro-apoptosis protein positively regulated the process of apoptosis, no significant elevation was observed. On the contrary, significant reduction were observed in treatment applying $1 \mu \mathrm{M}$ ART combined with $10^{-6} \mathrm{M}$ TAM.

\section{ART inhibits protective autophagy in TAM-R cells}

Accumulating evidences suggested that autophagy was deemed to induce antiestrogen resistance in breast cancer cells (26). Therefore, we also investigated if autophagy would reduce in TAM-R cells in the interference of ART treatment. Endogenous LC3 can be used to measure the induction of autophagy. It is suggested that as the first mammalian protein, LC3 is specifically recruited to autophagosome membranes and involved in the formation of autophagosome. Beclin-1 is one of the critical markers responsible for both autophagosome formation and autolysosome fusion. Western blotting analysis revealed that ART did not reduce the protein levels of LC3 and Beclin-1, suggesting that ART could not inhibit autophagy in TAM-R cells (Figure 3). AMPK, a highly conserved Ser/Thr protein kinase balancing energy homeostasis and metabolic stress and positively mediating autophagy, was also tested. Results showed that high-dose $(3 \mu \mathrm{M})$ ART caused increased pAMPK, indicating that ART might conversely induce autophagy (Figure 3).

The above data suggested that ART had no influence on autophagy when combined with TAM. This impression was confirmed by fluorescent microscopy of autophagy (Figure 4). In this experiment, two positive controls were included: rapamycin, an autophagy inducer, and chloroquine, an autophagy inhibitor acting at 

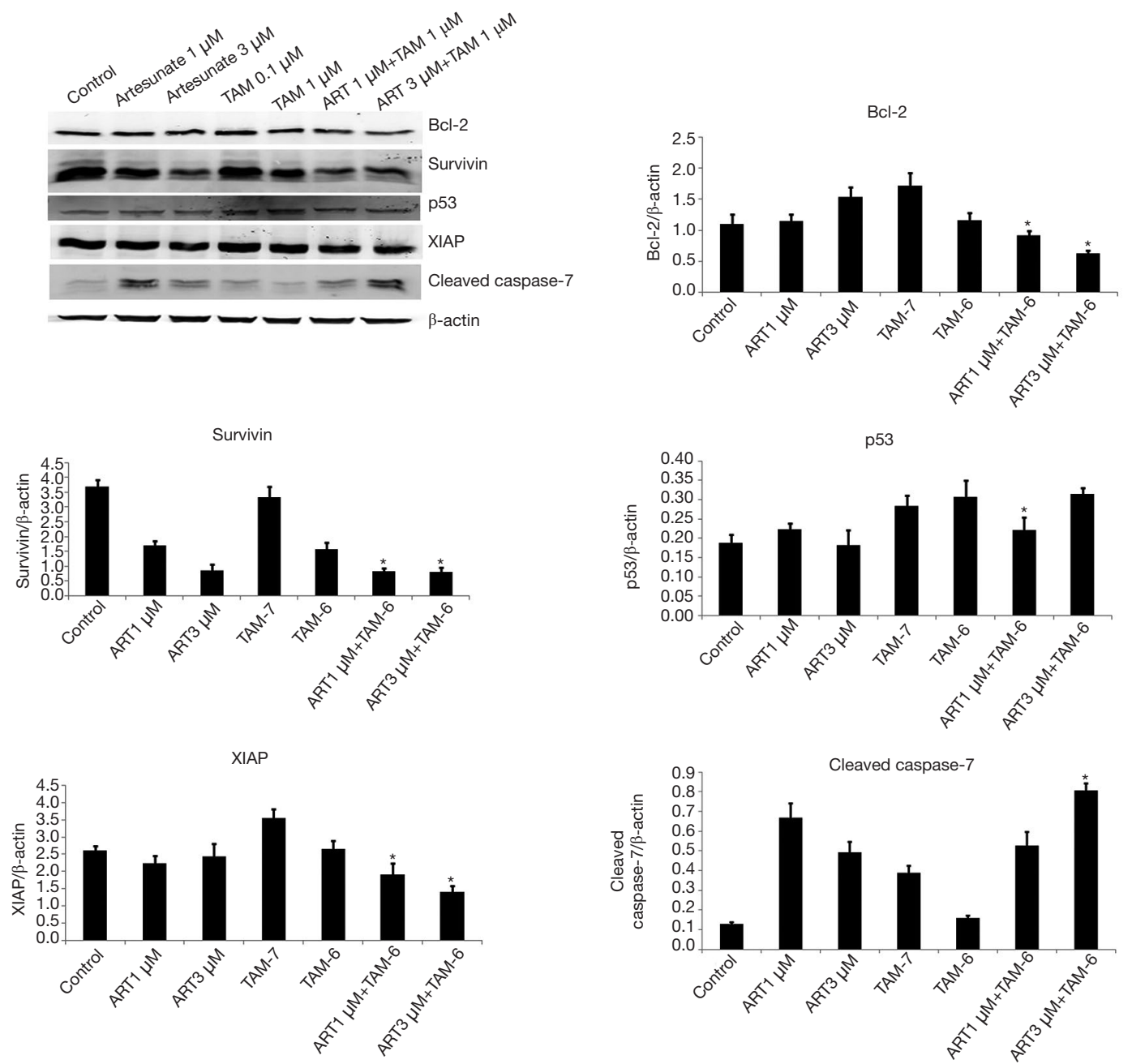

Figure 2 Artesunate therapy altered the expression of apoptosis markers Bcl-2, survivin, X-linked inhibitor of apoptosis protein (XIAP), p53 and cleaved caspase-7 in tamoxifen-resistant breast cancer cells (TAM-R) cells. TAM-R cells were plated in 60-mm dishes with 5\% fetal bovine serum (FBS) Improved Minimum Essential Medium (IMEM), and treated with artesunate (1 and $3 \mu \mathrm{M})$, tamoxifen (10 ${ }^{-7}$ and $\left.10^{-6} \mathrm{M}\right)$, and artesunate plus tamoxifen in culture medium for 72 hours when cells were about $80 \%$ of confluence before western blotting. Compared with $10^{-6} \mathrm{M}$ TAM, *, $\mathrm{P}<0.05$.

autophagolysosome. As shown in Figure 4, rapamycin increased the number of green punctate, autophagosomes. Green punctate were more and coarser in chloroquinetreated cells because autophagy flux was blocked. There was a moderate increase in the number of green punctate in the cells treated with TAM or Art alone. No significant change was seen when TAM and ART was combined.

\section{Discussion}

ART, approved by the US Food and Drug Administration, is hereto the most potent antimalarial drug available (27).
It is known for its safe and well-tolerated characteristics as it can selectively kill the cancer cells without harming the normal cells, and even can be applied in the fetus during the first trimester of pregnancy (28). In the year 1996, ART had already been suggested to possess ability in inducing apoptosis in human KG-1a leukemic cells (14). Since then, numerous trials on ART were conducted and revealed that ART could induce apoptosis in various cell types through different mechanisms $(29,30)$.

Apoptosis is a form of programmed cell death that occurs in multicellular organisms. Many pathways can trigger apoptosis. To investigate the mechanism of apoptosis 


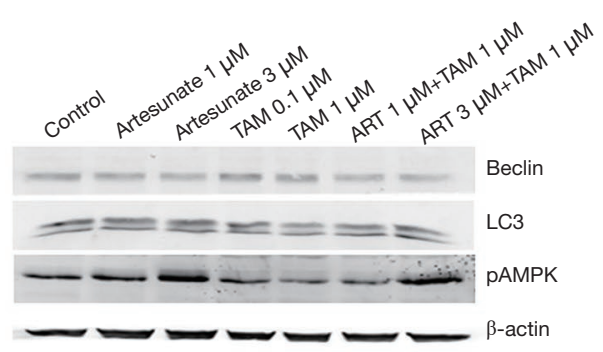

Beclin

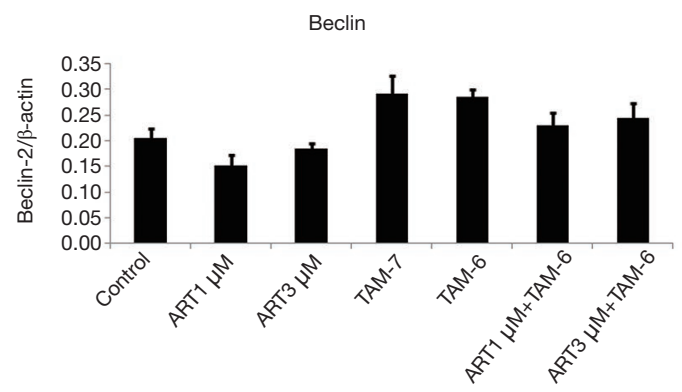

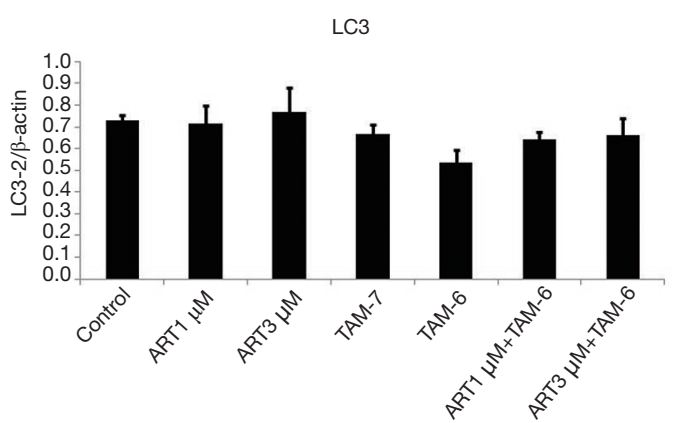

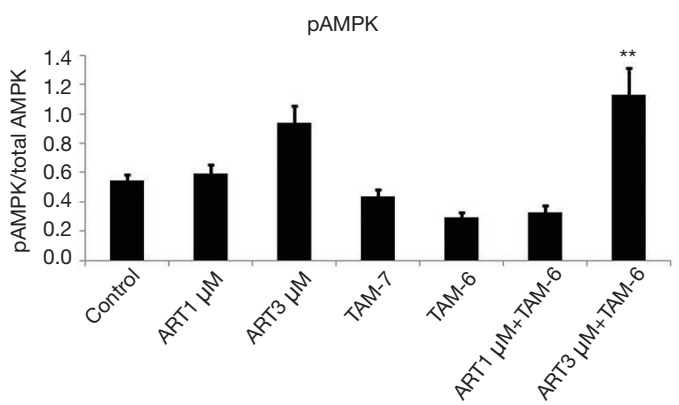

Figure 3 Artesunate therapy altered the expression of autophagy markers Beclin-1, LC3, and pAMPK in tamoxifen-resistant breast cancer cells (TAM-R) cells. TAM-R cells were plated in 60-mm dishes with $5 \%$ fetal bovine serum (FBS) Improved Minimum Essential Medium (IMEM), and treated with artesunate $(1$ and $3 \mu \mathrm{M})$ tamoxifen $\left(10^{-7}\right.$ and $\left.10^{-6} \mathrm{M}\right)$, and artesunate plus tamoxifen in culture medium for 72 hours when cells were about $80 \%$ of confluence before Western blotting. Compared with $10^{-6} \mathrm{M}$ TAM, ${ }^{* *}, \mathrm{P}<0.01$.

induced by ART in TAM-R cells, we evaluated several antiand pro-apoptotic proteins through western blot analysis. Bcl-2, Survivin, and XIAP proteins were shown to be significant decreased when TAM-R cells were treated with ART plus TAM, while caspase-7 was elevated significantly. Bcl-2 is an antiapoptotic protein that exhibits oncogenic potential through its ability to regulate the intrinsic apoptotic pathway. In breast cancer, evidences suggested that increased $\mathrm{Bcl}-2$ expression was associated with the estrogen receptor, increased histological grade and distant metastases (31), and correlated with chemotherapeutic and radiation resistance $(31,32)$. Our study detected decreased Bcl-2 expression in the use of ATR with TAM, suggesting that ART might possess ability to promote apoptosis in TAM-R cells.

The mechanism of apoptosis is executed with a cascade of sequential activation of initiator and effector caspases. Caspase-7, as one of the effector caspases, is an executioner protein of apoptosis. The activation of caspase-7 is considered a universal event associated with apoptosis induced by multiple stimuli. Inhibitor of apoptosis protein (IAPs) play evolutionary roles in the regulation of programmed cell death and apoptosis. Among the member of IAPs, XIAP has the most potent anti-apoptotic in cells, as it can directly inhibit the subset of cell death proteases (33). It was proven by previous studies that XIAP was an effective inhibitor of apoptotic stimuli that depended on caspase-7 (34). Results from our study showed that ART with TAM treatment reduced XIAP while significantly increased the expression of caspase-7, indicating that ART might trigger caspase-dependent apoptosis in TAM-R cells. The change in the Survivin levels further proved the above result. Survivin, like XIAP, is another member of the IAPs family of antiapoptotic protein. Survivin binds specifically to the terminal effector caspase-7 to protect cells from apoptosis (35). In our study, ART reduced Survivin levels which further proved that ART was a potent to inducer of apoptosis in TAM-R cells.

For $\mathrm{ER}^{+}$breast cancer patients, TAM remains the first-line adjuvant therapy. However, the acquisition of TAM resistance still is the biggest obstacle lie in the way of successful treatment. Numerous mechanisms are responsible for acquiring resistance toward TAM therapy, and autophagy is considered to be one of the mechanisms 


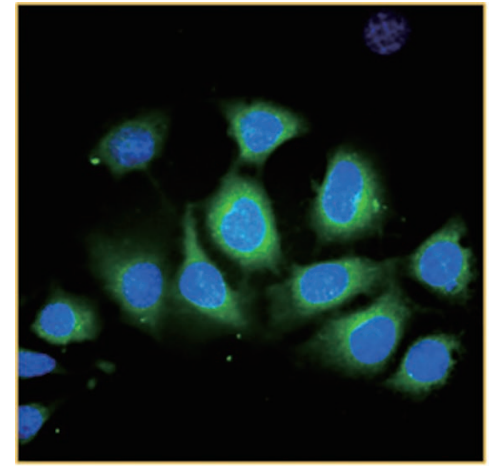

Control

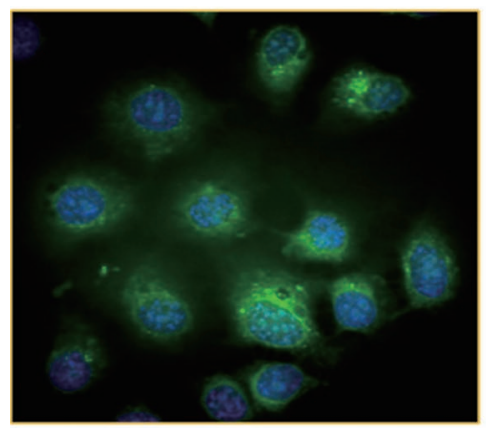

$\operatorname{Tam} 10^{-6} \mathrm{M}$

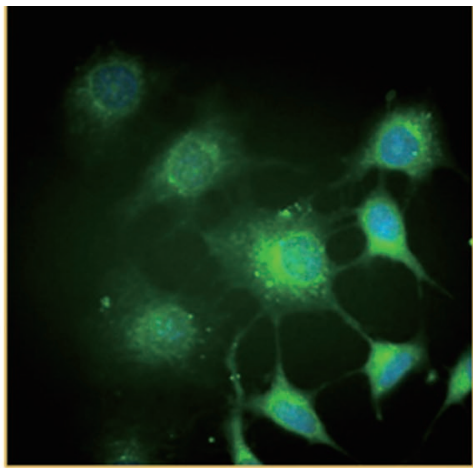

Rapamycin $20 \mathrm{nM}$ (positive control)

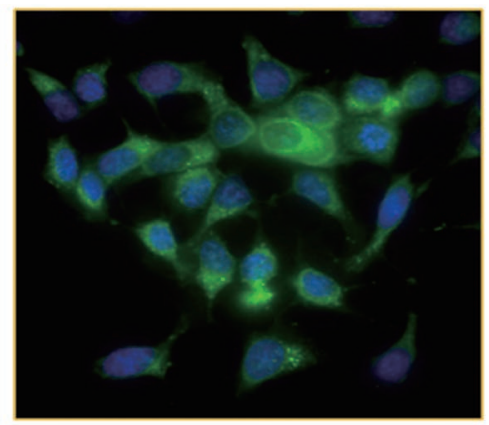

Artensuate $0.5 \mu \mathrm{M}$

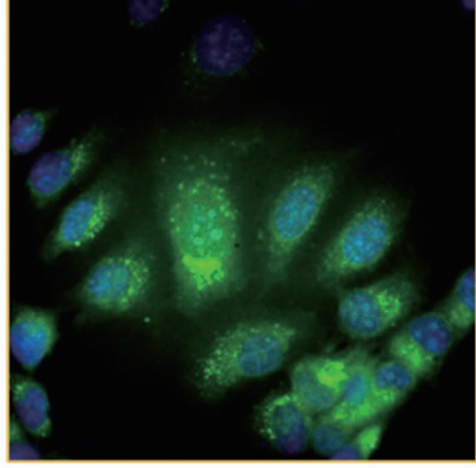

Chloroquine $10 \mu \mathrm{M}$ (positive control)

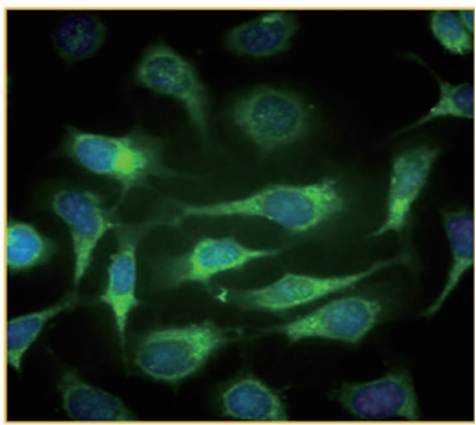

Tam+artensunate $0.5 \mu \mathrm{M}$

Figure 4 Autophagy fluorescent microscopy of tamoxifen-resistant breast cancer cells (TAM-R) cells. TAM-R cells grown on cover slip in 6-well plates in Improved Minimum Essential Medium (IMEM) - 5\% fetal bovine serum (FBS), and treated with testing agents for 24 h. TAM $10^{-6} \mathrm{M}$ (slightly increased autophagosomes), Artensuate $0.5 \mu \mathrm{M}$ (no obvious effect), tam + artensunate $0.5 \mu \mathrm{M}$ (still have autophagous bodies).

which is getting more attention in the recent years. Autophagy is an intracellular degradation process that is important in normal development and cellular response to metabolic stress. It can act as both tumor suppressor and protector. While the tumor-suppressive functions of autophagy mostly manifest at tumor initiation (36), the protective effect of autophagy becomes more apparent when tumor cells cope with microenvironmental stress (37). In environmental stimuli (high temperature, starvation, and low oxygen) or intracellular stresses (damaged organelles, accumulation of mutant proteins, and microbial invasion), autophagy is activated which results in survival of cancer cells in these severe environment (38). Previous investigations revealed that protective and pro-survival autophagy occurred in TAM-R cells $(8,39)$. An amount of studies had been successfully repressed autophagy in TAM-R cells by regulating certain proteins or micro RNA (40-42). In our study, we tried to explore whether the use of ART would inhibit autophagy and re-sensitize TAM-R cells to TAM therapy. LC3 is a key protein responsible for the major steps of autophagy. It is mainly localized in the nucleus, however, it functions primarily in the cytoplasm where the autophagosomes and autolysosomes arise (43), and determines autophagosome size and membrane curvature (39). Western blotting analysis showed that both low-dose and high-dose ART alone or combined with TAM did not significantly alter the LC-3 expressions in TAM-R cells, indicating that ART might have no effect on autophagy. The result of Beclin-1 further approve this negligible effect of ART on autophagy. Beclin-1, as the first identified autophagic gene in mammalian, can stimulate the formation of autophagosomes. Evidence showed that Beclin-1 involved in every major step in the autophagy pathway, from autophagosome formation to autophagosome/endosome maturation $(44,45)$. Western blotting analysis showed that ART treatment slightly reduced the expressions of Beclin-1, however, no significant difference was reached compared with controls, which 
denied the effect of ART in inhibiting protective autophagy. Results comparing the protein levels between ART plus TAM groups with TAM groups suggested that ART might not possess the ability to re-sensitize TAM-R to TAM. The measurement of pAMPK expressions further proved that ART might conversely induce autophagy.

Previous studies reported that the anticancer effects of ART were expressed through an apoptosis or autophagy pathway $(19,25)$. Our study focused on whether ART could induce apoptosis and inhibit autophagy in TAM-R cells, and if TAM-R cells could re-sensitize to TAM therapy through the intervention of ART. Increased apoptosis activity was detected when TAM-R cells were treated with ART and TAM in combination. However, little was found in ART on autophagy of TAM-R cells. The existed limitation of our experiment might be the reason of undesirable results. As ART inhibits the cell viability in a time- and dosedependent manner, we only investigated the effects of $1 \mu \mathrm{M}$ and $3 \mu \mathrm{M}$ ART on TAM-R cells, which might be insufficient to draw definite conclusion. Moreover, different sources of drug, antibody and assay equipment might also bring about inconsistent results.

\section{Conclusions}

The intervention of ART could not inhibit protective autophagy in TAM-R, however, possessed potential in inducing apoptosis. In addition, co-administration of 1 and $3 \mu \mathrm{M}$ of ART with $10^{-6} \mathrm{M}$ TAM might not be enough to resensitize TAM-R cells to TAM therapy.

\section{Acknowledgments}

Funding: The study is supported by Young Scientists Fund of the National Natural Science Foundation of China (No. 81503302), and 1166 Development Program for Junior Scientists, Dongfang Hospital affiliated to Beijing University of Traditional Chinese Medicine.

\section{Footnote}

Conflicts of Interest: All authors have completed the ICMJE uniform disclosure form (available at http://dx.doi. org/10.21037/tcr.2019.08.41). The authors have no conflicts of interest to declare.

Ethical Statement: The authors are accountable for all aspects of the work in ensuring that questions related to the accuracy or integrity of any part of the work are appropriately investigated and resolved. This study only involved cell lines analysis. The authors did not perform any experiments on human participants. Analysis did not involve the collection, use, or transmittal of individual identifiable data. As such, Institutional Review Board (IRB) approval to conduct this study was not required.

Open Access Statement: This is an Open Access article distributed in accordance with the Creative Commons Attribution-NonCommercial-NoDerivs 4.0 International License (CC BY-NC-ND 4.0), which permits the noncommercial replication and distribution of the article with the strict proviso that no changes or edits are made and the original work is properly cited (including links to both the formal publication through the relevant DOI and the license). See: https://creativecommons.org/licenses/by-nc-nd/4.0/.

\section{References}

1. Jemal A, Siegel R, Xu J, et al. Cancer statistics, 2010. CA Cancer J Clin 2010;60:277-300.

2. Montero AJ, Eapen S, Gorin B, et al. The economic burden of metastatic breast cancer: a U.S. managed care perspective. Breast Cancer Res Treat 2012;134:815-22.

3. Vergne Y, Matta J, Morales L, et al. Breast Cancer and DNA Repair Capacity: Association With Use of Multivitamin and Calcium Supplements. Integr Med (Encinitas) 2013;12:38.

4. Siegel RL, Miller KD, Jemal A. Cancer statistics, 2016. CA Cancer J Clin 2016;66:7-30.

5. Cardoso F, Bischoff J, Brain E, et al. A review of the treatment of endocrine responsive metastatic breast cancer in postmenopausal women. Cancer Treat Rev 2013;39:457-65.

6. Mandlekar S, Kong ANT. Mechanisms of tamoxifeninduced apoptosis. Apoptosis 2001;6:469-77.

7. Riggins RB, Schrecengost RS, Guerrero MS, et al. Pathways to Tamoxifen Resistance. Cancer Lett 2007;256:1-24.

8. Viedma-Rodríguez R, Baiza-Gutman L, SalamancaGómez F, et al. Mechanisms associated with resistance to tamoxifen in estrogen receptor-positive breast cancer (review). Oncol Rep 2014;32:3.

9. Yang ZJ, Chee CE, Huang S, et al. The Role of Autophagy in Cancer: Therapeutic Implications. Mol Cancer Ther 2011;10:1533-41.

10. Houben MP, Aben KK, Teepen JL, et al. Stable incidence 
of childhood and adult glioma in The Netherlands, 19892003. Acta Oncol 2006;45:272-9.

11. Abedin MJ, Wang D, Mcdonnell MA, et al. Autophagy delays apoptotic death in breast cancer cells following DNA damage. Cell Death Differ 2007;14:500-10.

12. Qadir MA, Kwok B, Dragowska WH, et al. Macroautophagy inhibition sensitizes tamoxifen-resistant breast cancer cells and enhances mitochondrial depolarization. Breast Cancer Res Treat 2008;112:389-403.

13. Efferth T, Kaina B. Toxicity of the antimalarial artemisinin and its dervatives. Crit Rev Toxicol 2010;40:405.

14. Efferth T, Rucker G, Falkenberg M, et al. Detection of apoptosis in KG-1a leukemic cells treated with investigational drugs. Arzneimittelforschung 1996;46:196-200.

15. Wang ZC, Liu Y, Wang H, et al. Research on the relationship between artesunate and Raji cell autophagy and apoptosis of Burkitt's lymphoma and its mechanism. Eur Rev Med Pharmacol Sci 2017;21:2238.

16. Kim C, Lee JH, Kim SH, et al. Artesunate suppresses tumor growth and induces apoptosis through the modulation of multiple oncogenic cascades in a chronic myeloid leukemia xenograft mouse model. Oncotarget 2015;6:4020-35.

17. Jeong DE, Song HJ, Lim S, et al. Repurposing the antimalarial drug artesunate as a novel therapeutic agent for metastatic renal cell carcinoma due to its attenuation of tumor growth, metastasis, and angiogenesis. Oncotarget 2015;6:33046-64.

18. Zhang LX, Liu ZN, Ye J, et al. Artesunate exerts an antiimmunosuppressive effect on cervical cancer by inhibiting PGE2 production and Foxp3 expression. Cell Biol Int 2014;38:639-46.

19. Chen K, Shou LM, Lin F, et al. Artesunate induces G2/ $M$ cell cycle arrest through autophagy induction in breast cancer cells. Anti-Cancer Drugs 2014;25:652-62.

20. Roh JL, Kim EH, Jang H, et al. Nrf2 inhibition reverses the resistance of cisplatin-resistant head and neck cancer cells to artesunate-induced ferroptosis. Redox Biology 2017;11:254-62.

21. Li PC, Lam E, Roos W, et al. Artesunate derived from traditional Chinese medicine induces DNA damage and repair. Cancer Res 2008;68:4347-51.

22. Dell'Eva R, Pfeffer U, Vené R, et al. Inhibition of angiogenesis in vivo and growth of Kaposi's sarcoma xenograft tumors by the anti-malarial artesunate. Biochem Pharmacol 2004;68:2359-66.

23. Steinbrück L, Pereira G, Efferth T. Effects of artesunate on cytokinesis and $\mathrm{G}_{2} / \mathrm{M}$ cell cycle progression of tumour cells and budding yeast. Cancer Genomics Proteomics 2010;7:337-46.

24. Huan-huan C, Li-Li Y, Shang-Bin L. Artesunate reduces chicken chorioallantoic membrane neovascularisation and exhibits antiangiogenic and apoptotic activity on human microvascular dermal endothelial cell. Cancer Lett 2004;211:163-73.

25. Tran TH, Nguyen AN, Kim JO, et al. Enhancing activity of artesunate against breast cancer cells via inducedapoptosis pathway by loading into lipid carriers. Artif Cells Nanomed Biotechnol 2016;44:1979.

26. Samaddar JS, Gaddy VT, Duplantier J, et al. A role for macroautophagy in protection against 4-hydroxytamoxifen-induced cell death and the development of antiestrogen resistance. Mol Cancer Ther 2008;7:2977-87.

27. Rosenthal PJ. Artesunate for the treatment of severe falciparum malaria. N Engl J Med 2008;358:1829-36.

28. Clark RL. Embryotoxicity of the artemisinin antimalarials and potential consequences for use in women in the first trimester. Reprod Toxicol 2009;28:285-96.

29. Li S, Xue F, Cheng Z, et al. Effect of artesunate on inhibiting proliferation and inducing apoptosis of SP2/0 myeloma cells through affecting NFkappaB p65. Int J Hematol 2009;90:513-21.

30. Ramacher M, Umansky VT. Effect of artesunate on immune cells in ret-transgenic mouse melanoma model. Anti-Cancer Drugs 2009;20:910.

31. Won KY, Kim GY, Kim YW, et al. Clinicopathologic correlation of beclin-1 and bcl-2 expression in human breast cancer. Hum Pathol 2010;41:107-12.

32. Kitada S, Takayama S, De RK, et al. Reversal of chemoresistance of lymphoma cells by antisense-mediated reduction of bcl-2 gene expression. Antisense Res Dev 1994;4:71-9.

33. Suzuki Y, Nakabayashi Y, Nakata K, et al. X-linked Inhibitor of Apoptosis Protein (XIAP) Inhibits Caspase-3 and -7 in Distinct Modes. J Biol Chem 2001;276:27058-63.

34. Deveraux QL, Takahashi R, Salvesen GS, et al. X-linked IAP is a direct inhibitor of cell-death proteases. Nature 1997;388:300-4.

35. Tamm I, Wang Y, Sausville E, et al. IAP-Family Protein Survivin Inhibits Caspase Activity and Apoptosis Induced by Fas (CD95), Bax, Caspases, and Anticancer Drugs. Cancer Res 1998;58:5315.

36. Debnath J. The Multifaceted Roles of Autophagy In 
Tumors--Implications For Breast Cancer. J Mammary Gland Biol Neoplasia 2011;16:173.

37. Roy S, Debnath J. Autophagy and Tumorigenesis. Semin Immunopathol 2010;32:383-96.

38. Bao XX, Xie BS, Li Q, et al. Nifedipine induced autophagy through Beclin1 and mTOR pathway in endometrial carcinoma cells. Chin Med J (Engl) 2012;125:3120-6.

39. Cook KL, Shajahan AN, Clarke R. Autophagy and endocrine resistance in breast cancer. Expert Rev Anticancer Ther 2011;11:1283.

40. Park JH, Ahn MY, Kim TH, et al. A new synthetic HDAC inhibitor, MHY218, induces apoptosis or autophagyrelated cell death in tamoxifen-resistant MCF-7 breast cancer cells. Invest New Drugs 2012;30:1887-98.

Cite this article as: Ding X, Yue W, Chen H. Effect of artesunate on apoptosis and autophagy in tamoxifen resistant breast cancer cells (TAM-R). Transl Cancer Res 2019;8(5):18631872. doi: $10.21037 /$ tcr.2019.08.41
41. Yu X, Luo A, Liu Y, et al. MiR-214 increases the sensitivity of breast cancer cells to tamoxifen and fulvestrant through inhibition of autophagy. Mol Cancer 2015;14:208-16.

42. Qi H, Jiang Z, Wang C, et al. Sensitization of tamoxifenresistant breast cancer cells by $Z$-ligustilide through inhibiting autophagy and accumulating DNA damages. Oncotarget 2017;8:29300-17.

43. Huang R, Liu W. Identifying an essential role of nuclear LC3 for autophagy. Autophagy 2015;11:852-3.

44. Kang R, Zeh HJ, Lotze MT, et al. The Beclin 1 network regulates autophagy and apoptosis. Cell Death Differ 2011;18:571.

45. He C, Levine B. The Beclin 1 interactome. Curr Opin Cell Biol 2010;22:140-9. 